\title{
Sustitución de Importaciones en la Industria Biofarmacéutica Argentina: Una Estrategia con Blanco Móvil
}

\author{
Pablo Lavarello ${ }^{1 *}$, Evelin Goldstein ${ }^{2}$, Juan José Pita ${ }^{3}$
}

Resumen: Desde una perspectiva de sustitución de importaciones basada en la industria infantil, la protección del mercado interno es necesaria como un primer paso para generar un proceso de aprendizaje tecnológico. Esta perspectiva es de relevancia frente a la emergencia de la biotecnología como paradigma tecnológico. Para ciertos países en desarrollo como la Argentina que han alcanzado tempranamente un umbral mínimo de conocimientos y ciertas capacidades manufactureras, esto abre una ventana de oportunidad. Este trabajo muestra a partir de datos de comercio la existencia de espacio para sustitución de importaciones dependiendo de la complejidad de las tecnologías y discute ciertos aspectos para impulsar deliberadamente este tipo de estrategias.

Palabras clave: sustitución de importaciones; capacidades tecnológicas infantiles; biofármacos.

Import Substitution in Argentine Biopharmaceutical Industry: A Moving Target Strategy

Abstract: From an infant capability approach of import substitution, domestic market protection is a necessary first step to generate technological learning. This view is relevant in the face of biotechnology paradigm emergence. For developing countries as Argentina, that had early achieved a minimum threshold of knowledge and manufacturing experience, this opens a window of opportunity. This works shows that there is space for an importation substitution strategy in Argentina depending on the complexity of technologies involved discussing some aspects to encourage deliberately this strategy.

Keywords: import substitution; infant technology capabilities; biopharmaceutical products.

Submitted: May 30 $2016 /$ Approved: November March $7^{\text {th }} 2017$

\section{Introducción ${ }^{1}$}

Este trabajo se propone discutir las posibilidades para los países de América Latina para avanzar en un proceso de sustitución de importaciones en industrias de alta tecnología como lo es la industria de biofármacos. Industria en la que un conjunto acotado de firmas han desarrollado capacidades de exportación y en los cuales su complementación con mecanismos de protección selectiva del mercado interno pueden llegar a reducir el déficit comercial.

La sustitución de importaciones ha sido una estrategia implementada en diversos países en desarrollo, particularmente en América Latina y en Asia. Sin embargo, en algunos casos, la sustitución de importaciones ha sido un fin en sí mismo, con el objetivo de solucionar la falta de oferta de productos en el mercado interno y enfrentar los problemas de restricción de divisas, mientras que en otros ha constituido una pieza de la política de industrialización (Ocampo, 2009; Thorp,1998). Ha sido ampliamente estudiado cómo estrategias de sustitución de importaciones han tenido momentos de apogeo y de declive. Es por ello que la sustitución de importaciones ha sido objeto de críticas y controversias.
Al respecto, incluso Prebisch, que destaca el impulso a la transformación estructural que ha provisto la sustitución de importaciones, también reconoce que se ha incurrido en graves fallas, esencialmente por dos razones. La primera, porque no se han seguido consideraciones de economicidad, por lo cual no se ha logrado una reducción del coeficiente de importaciones; y la segunda, porque en algunos casos no se ha alentado complementariamente las exportaciones. De esta manera, propone ir más allá de la dicotomía planteada por algunos autores entre la sustitución de importaciones y la promoción de exportaciones como estrategia de desarrollo industrial.

El punto esencial que es importante remarcar, que ciertos autores enfatizan, es que la protección del mercado interno es necesaria como un primer paso para generar un proceso de aprendizaje tecnológico que permita a las empresas consolidar su posicionamiento competitivo (Kim, 1990; Ray, 1998). En línea con la literatura de la protección a la industria infantil inspirada en los trabajos de Frederich List y Alexander Hamilton, la sustitución de importaciones deja de ser un fin en sí mismo. La protección selectiva y transitoria del mercado interno posibilita el desarrollo de capacidades tecnológicas. Como lo demuestran los trabajos de Amsden $(1985,1995)$ y Kim (1990) basados en las experiencias de catching up de Korea y Taiwan, la protección a las capacidades tecnológicas infantiles fue el principal móvil de las experiencias de sustitución de importaciones en esos países.

(1) CEUR-CONICET, Saavedra 15, 1083ACA, CABA, Argentina

(2) Idear Desarrollo, Saavedra 15, 1083ACA, CABA, Argentina

(3) UBA, Saavedra 15, 1083ACA, CABA, Argentina

Autor de correspondencia: plavarello@conicet.gov.ar

(1) Este trabajo presenta algunos de los resultados del proyecto PICT 2034 "Estrategias de inserción internacional de la industria farmacéutica frente a la biotecnología: el caso de la Argentina” desarrollado en el CEUR-CONICET 
En el caso particular de la experiencia de sustitución de importaciones en Argentina entre los años 50 y 70 la producción de bienes finales de tecnología media no logró avanzar en un proceso acelerado de protección a las capacidades tecnológicas infantiles. Los esfuerzos tecnológicos se limitaban a la adaptación de procesos y ajustes de lay outs de plantas con un alto grado de integración de componentes. Si bien la adaptación tecnológica tardía posibilitó aprendizajes de proceso, fue generando con su desarrollo niveles de protección cada vez más altos para compensar la ampliación de las brechas tecnológicas en las industrias de insumos y componentes ${ }^{2}$.

La introducción de la protección infantil de las capacidades tecnológicas como dimensión central en la estrategia de sustitución de importaciones plantea como interrogantes cómo pueden aprovecharse las ventanas de oportunidad y en que momento en el marco de una estrategia de este tipo. Mientras que ciertos autores plantean explícita o implícitamente que las posibilidades de catching up son mayores en la madurez de las tecnologías apostando a avanzar rápidamente en los procesos de aprendizaje en la producción, otros autores plantean que las posibilidades son mayores en los momentos iniciales de difusión de una nueva tecnología cuando los parámetros de los procesos de manufactura aún no se han consolidado y las empresas de los países periféricos pueden avanzar en el desarrollo imitativo de productos, componentes y procesos (Amsden, 2004; Perez y Soete, 1988).

Esta pregunta es de relevancia para ciertos países en desarrollo como es el caso de Brasil y Argentina quehan alcanzado tempranamente un umbral mínimo de conocimientos en biología molecular y ciertas capacidades de bioprocesamiento, en particular en el desarrollo y manufactura de productos biofarmáceuticos. A diferencia dela industria farmacéutica tradicional en los que dada la alta madurez de las tecnologías de síntesis química y las elevadas economías de escala hacen quela sustitución de importaciones de los insumos básicos no sea rentable, las bajas barreras de escala y experiencia requerida al inicio de la difusión de las biotecnologías abrirían una nueva ventana de oportunidad (Gutman y Lavarello, 2014).

Sin embargo, diversos trabajos internacionales realizados por especialistas del sector han relativizado las ventanas de oportunidad señalando las dificultades que puede implicar este tipo de estrategia dada la alta complejidad de los productos biológicos y sus procesos de producción y aprobación regulatoria (Moorkens, et al, 2016; Rader, 2013). Aspecto que es reforzado por el continuo surgimiento de oleadas de nuevos conocimientos y productos plantea nuevas barreras de conocimiento a tener en cuenta en este tipo de estrategias (Lavarello, 2014).

Este trabajo se plantea como interrogante exploratorio en qué medida dichas oportunidades han posibilitado procesos de sustitución de importaciones en productos farma-biotecnológicos en Argentina buscando identificar si las ventanas son transitorias o si existieron segmentos de productos en los que este tipo de estrategias fueron efectivas.
A fin de responder a este interrogante en la sección 1 desarrollamos, a partir de una revisión de aportes de los autores neoschumpeterianos, un marco conceptual que permita analizar cuáles son las barreras que enfrenta un país para entrar en un sector de alta tecnología a partir de una estrategia de sustitución de importaciones. En la sección 2 se analiza cómo evolucionó el déficit de biofármacos en Argentina durante los últimos 16 años. En la sección 3 se realiza un ejercicio exploratorio que busca identificar preliminarmente cuáles son los productos con posibilidad de sustitución de importaciones, discutiendo cuales son las eventuales barreras que pueden impedir ese proceso. En las conclusiones se presentan los resultados, sus implicancias para la política pública y los nuevos interrogantes para investigaciones futuras.

\section{Marco conceptual: la sustitución de importaciones frente a los nuevos paradigmas tecnológicos.}

Mientras que América Latina desmantelaba en los años '80 sus políticas basadas en la sustitución de importaciones en sectores maduros, diversos países de Asia llevaban adelante un proceso de sustitución de importaciones en sectores de alta tecnología (Amsden, 2004). El éxito de la estrategia se basó, esencialmente, en el impulso de la producción imitativa de bienes de alta tecnología que estaban por entrar a su fase de madurez y a partir de allí avanzar a los más innovativos. Estrategia que se dio en simultáneo a la articulación de proveedores locales de las piezas e insumos. De esta manera, con la sustitución de importaciones de los bienes finales $\mathrm{y}$, en algunas de las etapas, de producción de sus componentes, se evitaba caer en un estrangulamiento de la balanza de pagos.

El avance en la sustitución de componentes, por un lado, y la complementación de políticas coordinadas de créditos subsidiados, creación de centros de innovación para transferencia tecnológica y la fijación de metas claras de producción e industrialización, por el otro, han sido las principales diferenciales de la política de Asia respecto de América Latina, que han permitido la continuidad de la estrategia y el éxito de la misma.

Cabe destacar el activo papel de los gobiernos en los procesos de sustitución de importaciones en los países asiáticos, con la aplicación de un mix de políticas específicas para la promoción de la producción de bienes de alta tecnología, que incluían incentivos a la generación de capacidades tecnológicas, por ejemplo, beneficios fiscales, créditos con tasas de interés subsidiadas, la creación de parques industriales científico-tecnológicos. También ha sido clave el rol de las instituciones científicas públicas en la investigación, desarrollo y diseño de nuevos productos como en el fortalecimiento de recursos humanos idóneos para trabajar en los nuevos segmentos de mercado (Cimoli et al, 2006).

Pero fundamentalmente estas experiencias estuvieron signadas por una alta selectividad de los sectores (y de las firmas) a través de múltiples instrumentos que incluían desde la producción pública de componentes clave hasta la organización del mercado asegurando

(2) Aún cuando la estrategia de industrialización comienza a ser corregida a partir del año 1964 a partir de la promoción de inversiones industria pesada (acero, petroquímica, aluminio, papel), las políticas de apertura comercial y financieras de fines de los años '70 abortan la emergencia de una incipiente industria de proveedores especializados de bienes de capital e industrias basadas en la ciencia (electrónica y farmoquímica) 
un adecuado mix entre competencia y protección (Amsden, 1992). En estos casos la estrategia se basó en inicialmente apoyar industrias de ensamble en productos que comenzaban su fase de madurez focalizando el aprendizaje acelerado en los procesos de manufactura de los componentes tecnológicamente intensivos. El estado impulsó la creación de infraestructuras de CyT que resolvieran los cuellos de botella tecnológicos e impulsar desprendimientos privados de laboratorios estatales.

\subsection{Las ventanas de oportunidad en el marco de los nuevos paradigmas} La selectividad de la política invita a reflexionar sobre cuáles es momento más adecuado del ciclo de los productos para comenzar la sustitución de importaciones. Los autores neoschumpeterianos analizan las condiciones bajo las cuales ciertos países en desarrollo han logrado entrar a una industria de alta tecnología frente a la emergencia de nuevos paradigmas tecnológicos (Perez y Soete, 1988: 458; Malerba y Nelson, 2011:1645).

Desde la perspectiva de la literatura del ciclo de producto (Vernon, 1966) la sustitución de importaciones en los países periféricos se da en el marco de la transferencia de la tecnología desde el centro cuando la misma ya ha alcanzado cierta madurez. Amsden (1992) señala a partir de los análisis de los sectores de semiconductores y los lectores de disco que las posibilidades de sustitución de importaciones son mayores cuando estos productos comienzan su fase de madurez. Es en esta fase que la competencia se centra en el aprendizaje acelerado.

Desde una perspectiva neo-shumpeteriana esta posición puede ser relativizada (Perez y Soete, 1988; Perez, 2002). En la medida que los productos maduros son justamente aquellos que han agotado su dinamismo tecnológico, la focalización de la sustitución de importaciones en los mismos puede generar un problema de "encerramiento" (Lock in) en un sendero de desarrollo de bajos salarios y bajo crecimiento. En línea con esta crítica del modelo de ciclo de producto plantean que los países semi-industralizados cuentan con posibilidades de entrada de las empresas de estos países en las etapas tempranas de difusión de la tecnología.

Perez y Soete (1988) plantean que en el momento de transición entre paradigmas tecnológicos, los países desarrollados han hundido inversiones y aprendizajes tecnológicos en las tecnologías preexistentes resultando en altas barreras a la salida. Esto implica que al inicio del ciclo de una nueva tecnología existirán posibilidades de ascenso industrial para aquellos países en desarrollo que generen la base de conocimiento en la nueva tecnología. Aún cuando la probabilidad de dicho proceso de ascenso industrial son limitadas, es de relevancia analizar las condiciones que los hicieron posibles ${ }^{3}$.

Es posible avizorar que las condiciones favorables para un proceso de sustitución de importaciones varía según la etapa de difusión de la tecnología. En la fase inicial de emergencia de una nueva tecnología hasta que la misma se consolida en un conjunto de parámetros y heurísticas las economías de escala son bajas, exigiendo en contraste un alto umbral de conocimientos para asimilar las nuevas tecnologías. En la fase de crecimiento, en la que el mercado ya se creó y se expande a alto ritmo, los parámetros de los procesos ya se encuentran definidos y los productos están ampliamente testeados en el mercado. El foco cambia de la I+D a los aprendizajes en la producción. El umbral requerido de capacidades en I+D disminuye y aumenta el umbral mínimo de experiencia en la producción. En la fase siguiente, el crecimiento del mercado comienza a desacelerarse y los procesos ya han sido optimizados. Las economías de escala aumentan. Las posibilidades de nuevos entrantes disminuyen aunque el acceso al conocimiento CyT es mayor. Finalmente, en la fase de madurez, ya se estandarizaron tanto el producto como el proceso. Ya las posibilidades de mejoras en la productividad son menores y la ventaja se encuentra asociada a contar con costos más bajos en las materias primas o en la mano de obra.

El análisis del ciclo de la industria permite concluir que las dos etapas en las que existen mayores posibilidades de entrada para las firmas de los países en desarrollo son la primera en la que emerge la tecnología, a partir de una inserción autónoma como imitadores tempranos, y la última como productores de bajo costo en el marco de las estrategias deslocalización de las Empresas Multinacionales. No obstante, los desafíos no son menores en el primer caso para los países en desarrollo, en la medida que la entrada como imitadores tempranos exige tanto un alto esfuerzo en I+D y una infraestructura de CyT que brinde el conjunto de externalidades necesarias para compensar las desventajas vis a vis las ventajas de las empresas multinacionales de los países desarrollados (y sus respectivos sistemas nacionales de innovación).

\subsection{Los ciclos de vida de los paradigmas tecnológicos: las especifi-} cidades de la biotecnología

El análisis para una tecnología particular puede ser ampliado para productos y tecnologías que forman parte de sistemas técnicos (Freeman y Perez 1988). En este sentido, las sucesivas tecnologías y productos que pueden ser comprendidos como mejoras incrementales en un mismo producto. Esta visión es altamente útil para el caso de las biotecnologías en la medida que la mismas comprenden el surgimiento de sucesivas oleadas tecnológicas - ingeniería genética, genómica, proteómica, bioingeniería de tejidos, otras- y sucesivas generaciones de productos que van dando lugar a la emergencia de un paradigma tecnológico.

Desde esta perspectiva cada nueva tecnología se beneficia del conocimiento y la experiencia de las tecnologías precedentes así como de la infraestructura de CyT desarrollada previamente. Pasamos a una dimensión analítica en la que estudiamos los ciclos de vida ya no de los productos sino de los paradigmas tecnológicos. Cuestión que tiene importantes implicancias para la economía del desarrollo. Aquellos países desarrollados en los que la base de conocimientos, los

(3) El logro de este este tipo de procesos se limita a unas pocas experiencias de industrialización tardía del siglo XIX como Estados Unidos, Alemania y Francia frente al declive de Gran Bretaña. Más recientemente Japón y Corea del Sur atravesaron experiencias similares en un conjunto de ramas entre las cuales la difusión de la micro-electrónica en procesos y productos, y más parte las TICs, lograron procesos de catching up exitosos. 
aprendizajes y la organización de sus sistemas nacionales de innovación aún se encuentran orientados hacia paradigmas tecnológicos previos pueden quedar encerrados (lock-in) en un sendero de desarrollo de bajo crecimiento.

No obstante, el caso de las biotecnologías es posible mencionar un conjunto de aspectos que deben ser consideradas a la hora de analizar las condiciones de entrada.

En primer lugar, los umbrales mínimos de $\mathrm{I}+\mathrm{D}$ no han disminuido como se esperaba al inicio de la difusión del paradigma. Las sucesivas oleadas de biotecnología no se han traducido necesariamente en una base de conocimientos común. Cada nueva oleada implica una ampliación de la base de conocimiento que aumenta la complejidad de la misma y aumenta los costos de I+D sin necesariamente resultar en rendimientos crecientes de la innovación (Pisano, 2002). Esto implica que el umbral mínimo de I+D no disminuya con la difusión del paradigma.

En segundo lugar, no es claro que el nuevo paradigma tecnológico reemplace necesariamente al paradigma preexistente en todas las aplicaciones de la biotecnología (Lavarello, 2014). En las aplicaciones de biotecnología industrial (Enzimas industriales e Ingredientes alimentarios) los bioprocesos son complementarios a tecnologías previas. Aún en las aplicaciones en biotecnología de salud en los que las técnicas y productos biotecnológicos han reemplazado los productos de síntesis química, muchos procesos en la I+D de una molécula requieren capacidades químicas y biológicas tradicionales.

En tercer lugar, no es claro en la literatura la tendencia al aumento en la escala coexistiendo producciones con escalas altas y bajas según el tipo de molécula. Si bien se manifestó un aumento en el requisito de escala - con grandes fermentadores de acero inoxidable $e^{4}$ y procesos en continuo -, durante los últimos años surgieron nuevas tecnologías que posibilitan producir de forma más flexible aprovechando economías de scope (variedad) 5 . Estas tecnologías si bien requieren menor inversión en grandes plantas y menores tiempos de ajuste al cambiar de producto, involucran mayores costos unitarios, siendo compatibles con una estrategia de inserción rápida en nichos para productos de ciclo de vida corto.

En cuarto lugar, la experiencia es un requisito crítico en la medida que se avanza de moléculas más simples a aquellas más complejas. La producción a escala industrial de productos biológicos es altamente variable. El logro de lotes de producción manteniendo la estabilidad biológica resulta clave para asegurar la eficacia y seguridad de la droga. Esta condición es más difícil de lograr en las moléculas más complejas como los anticuerpos monoclonales utilizados para tratamientos oncológicos y enfermedades crónicas de alto costo que en moléculas biotecnológicas de primera generación como los interferones.
Last but not least, las barreras regulatorias en los medicamentos asumen una importancia mayor que los otros determinantes. La entrada como imitadores tempranos en las biotecnologías de salud implica cumplir con aprobaciones sanitarias y del régimen de propiedad intelectual específicas a cada país. En aquellos casos en los que la altura inventiva requerida para patentar sea baja y el alcance de las reivindicaciones sea amplio las barreras a la entrada de nuevos jugadores será mayor. Por su parte el establecimiento de marcos regulatorios estrictos exigiendo a los imitadores replicar los largos y costosos ensayos clínicos frenará las posibilidades de aprobación de moléculas similares a las originales (Gutman y Lavarello, 2015). Frente a ello una estrategia de propiedad intelectual y regulatoria que permita generar los aprendizajes de las firmas para sortear las barreras es clave para los países seguidores.

En resumen, la sustitución de importaciones en el caso de los biofarmacéuticos requiere un conjunto de intervenciones que logren absorber rápidamente nuevas oleadas de conocimiento biotecnológico y fomentar la generación de capacidades tecnológicas en la manufactura acompañados de un manejo estratégico de la propiedad intelectual y de la aprobación sanitaria a medida que aumentan los umbrales regulatorios. Los mayores riesgos en dicho proceso de sustitución de importaciones es el de elegir la técnica de producción correcta y el riesgo de mercado.

\section{Industria biofarmacéutica Argentina durante los años 2000: expansión del mercado interno y déficit comercial creciente.}

El mercado de productos biofarmacéuticos en Argentina experimentó una acelerada expansión entre desde mediados de los años 2000. Mientras que en el año 2006 los biofármacos representaban el 11\% de las ventas totales de la industria farmacéutica, en el año 2013 estos llegaron a representar el $23 \%$ de dicho mercado. Esto muestra que los biofármacos constituyen una de las principales áreas de expansión del mercado farmacéutico.

Gráfico $\mathrm{N}^{\circ} \mathbf{1}$. Argentina: mercado local. Millones de pesos

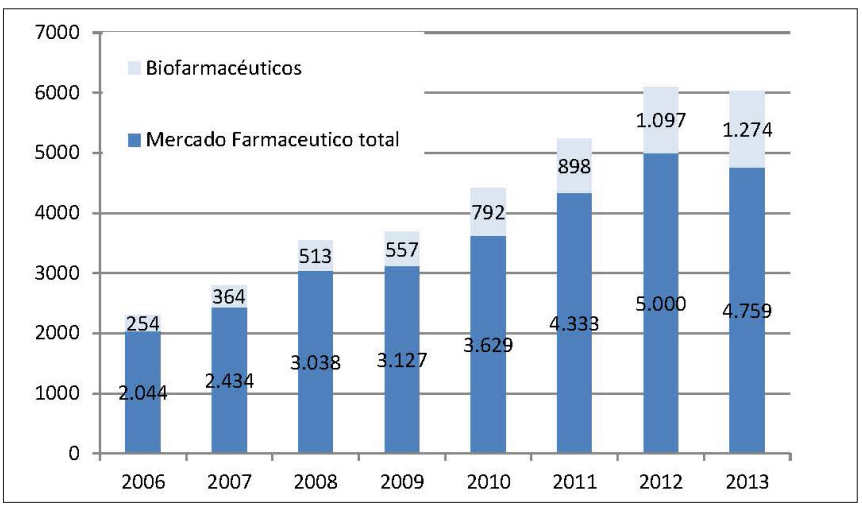

Fuente: elaboración propia a partir de datos de aduana y ministerio de economía

(4) No obstante existen trade offs entre la escala y el tiempo de bioproceso. En general los sistemas de producción de menor escala como los de perfusión requieren más tiempo de bioproceso. En contraste, los sistemas en batch o feed-batch son de mayor escala y menor tiempo de bioproceso.

(5) Se trata de sistemas de producción en bolsas descartables que posibilitan fabricar distintos productos en una misma planta disminuyendo los tiempos de limpieza y validación de la planta. Las bolsas tienen un alto costo y no pueden ser mayores a 1000 o 2000 litros. siendo compatibles con una estrategia de producción flexible. 
La producción de biofármacos en Argentina se distingue de otros mercados de productos biotecnológicos por la presencia de empresas privadas nacionales asociadas a los grandes grupos farmacéuticos que desarrollan y manufacturan localmente. A diferencia de lo que sugiere la teoría del ciclo de vida de los productos de Vernon (1966), estas firmas exportaron tempranamente productos biotecnológicos de primera generación aún sin contar con una presencia dominante en el merado interno (Gutman y Lavarello, 2015). La inserción como exportadores tempranos posibilitó para un reducido conjunto de firmas aprendizajes en la manufactura de biológicos a partir de escalas chicas de producción -con sistemas de fermentación de 500 a 1000 litros- e importantes aprendizajes junto a la autoridad regulatoria en un momento donde los umbrales de escala y regulatorios aún no eran críticos.

A pesar de dicha dinámica fuertemente exportadora de las firmas biofarmacéuticas argentinas, el mercado local se encuentra controlado por las empresas líderes a nivel internacional. Una primera lectura de la trayectoria comercial de productos biofarmacéuticos en la Argentina muestra su carácter deficitario, el cual se ha profundizado a lo largo de la última década. Mientras el saldo comercial del conjunto de moléculas biotecnológicas era de US\$ 50 millones en el año 1998, nueve años después superó los US\$ 600 millones. La razón es que las importaciones se han expandido a un ritmo mayor a las exportaciones en el período, acelerándose la diferencia a partir del año 2008 (ver Gráfico 1).

Gráfico 2. Evolución de las exportaciones e importaciones del sector salud. Miles de dólares

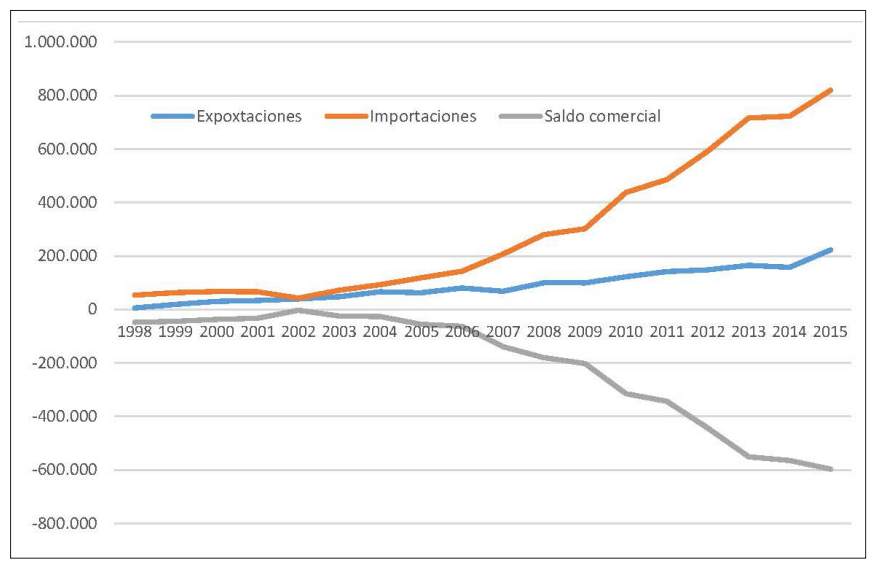

Fuente: elaboración propia en base a datos de ADUANA.

De este análisis agregado de la evolución del comercio exterior de los productos farmacéuticos, se obtienen, al menos, dos conclusiones. Por un lado, que las empresas locales han logrado consolidar su inserción como exportadoras de moléculas biotecnológicas; pero por el otro, en vistas de que las importaciones han mostrado un aumento vertiginoso, el sector biofarmacéutico argentino ha resultado deficitario y, año a año, profundiza dicho rasgo estructural de la industria, lo que invita a plantear la necesidad de observar la composición de dicho déficit, desagregando la evolución comercial según el tipo de producto.
Es posible apreciar en el gráfico $\mathrm{N}^{\circ} 2$ que el déficit del sector biofarmacéutico argentino se encuentra asociado a las moléculas más complejas -como los anticuerpos monoclonales denominados MABs por sus siglas en inglés, utilizados en enfermedades de alto costo para los sistemas de salud como las oncológicas- y otras moléculas recombinantes que no pudieron ser identificados y figuran en "los demás" pero estimamos que se trata también de moléculas complejas que no cuentan con una desagregación adecuada en el del nomenclador común del MERCOSUR (NCM). Además de estos, hay otros productos biofarmacéuticos que también tienen un saldo negativo en el comercio exterior, aunque poco significativos en relación al total dado su menor volumen total de comercio. Estos son productos menos complejos ya ampliamente difundidos (como es el caso de los interferones, somatotropina y la insulina y el Filgrastim peguilado). Por tanto, si bien no es el único conjunto con signo negativo, son los MABS los productos del sector de biofármacos los que explican el resultado agregado deficitario y creciente en la última década.

Gráfico 3. Saldo comercial promedio por grupo de productos. Miles de dólares

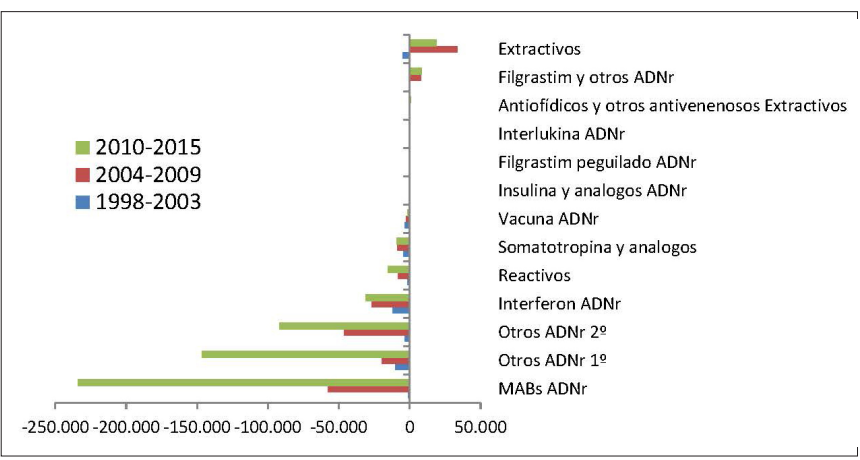

Fuente: elaboración propia en base a datos de ADUANA.

Por su parte, existe un conjunto de moléculas biotecnológicas y biológicas en los que la Argentina ha logrado insertarse mostrando superávits crecientes desde mediados de los años ' 90 . Este es el caso de numerosos productos biológicos extractivos, y drogas biotecnológicas correspondientes a la primera oleada de biotecnológicos como lo son los principios activos recombinantes de EPO y el filgrastim.

Apoyados en una larga trayectoria de producción de biológicos, un conjunto acotado de empresas locales se han insertado como exportadoras netas de estos productos. Mientras dos laboratorios nacionales explican el superávit en biológicos extractivos, otras tres empresas locales explican la inserción exportadora en las tres moléculas recombinantes de la primera generación. También existe un conjunto de proteínas recombinantes en los que si bien la Argentina aún es deficitaria, en los últimos años se ha logrado reducir el saldo negativo, como es el caso de las vacunas contra la hepatitis. Si bien se trata en todos los casos de moléculas de primera generación (recombinantes o extractivas) hoy ampliamente difundidas y con bajos márgenes de ganancia, estos resultados muestran que existe cierto potencial para la sustitución de importaciones de moléculas biotecnológicas y su inserción como exportador biotecnológico. 


\section{Las posibilidades de sustitución de importaciones en el sector de biofármacos}

Las cifras de la evolución del comercio exterior de los productos del sector de biofármacos demuestran que el déficit es significativo y creciente en los últimos años. En este sentido, cabe indagar acerca de la factibilidad de sustituir importaciones y mejorar su inserción externa.

\subsection{Un abordaje metodológico exploratorio.}

Para identificar en forma preliminar los productos en los que la Argentina tiene capacidades para producir localmente, se procedió, en primer lugar, con la identificación de las posiciones arancelaras a 12 dígitos de la Nomenclatura Común del Mercosur (NCM) correspondientes a los productos biotecnológicos ${ }^{6}$. Para dichas posiciones arancelarias se calculó el Índice Grubel-Lloyd (IGL), con el objetivo de clasificar los productos que actualmente presentan déficit comercial en función de su factibilidad de ser sustituidos. Este índice muestra el grado de comercio intra-industrial de cada rama. Asimismo, en aquéllas posiciones en las cuales existen exportaciones, a pesar de que sean deficitarias, es posible considerar la disponibilidad de cierta capacidad local de producción. Al trabajar con elevada desagregación del nomenclador - posiciones a 12 dígitos - se logra despejar en buena medida las situaciones en las que productos de una misma posición son muy diferentes en términos de capacidades requeridas, complejidad de las moléculas y grado de diferenciación de los productos. No obstante la existencia de posiciones no bien especificadas limita el grado de precisión de los resultados.

A partir de la estimación de estos indicadores, se construyeron cinco categorías, de acuerdo al valor obtenido de IGL para cada posición arancelaria, para reflejar diferentes grados de probabilidad de sustitución de importaciones (SI):
1) superavitarias (posiciones arancelarias con saldo comercial positivo);

2) alta probabilidad de SI (saldo comercial deficitario, con IGL entre 0,66 y menor o igual que 1 );

3) moderada probabilidad de SI (saldo comercial deficitario, con IGL, menor o igual que 0.66 y mayor que 0.33 );

4) potencial probabilidad de SI (saldo comercial deficitario, con IGL entre 0,09 y menor o igual que 0,33 )

5) baja probabilidad (saldo comercial deficitario, con IGL menor o igual que 0,09$)$.

De acuerdo a los resultados obtenidos para los tres subperíodos considerados, como puede apreciarse en el Cuadro $\mathrm{N}^{\circ} 1$, los productos biotecnológicos superavitarios estaban representados por 7 posiciones arancelarias durante 1998-2003 y pasaron a ser9durante 2004-2009 y 13 en 2010-2014 reflejando la capacidad de la industria de ampliar su cartera de productos. Esto se refleja, además, en un aumento del superávit promedio anual de estos productos que pasó de representar solo U\$S 16 millones a 100 U\$S millones. Asimismo, se observa que existen actualmente 2 posiciones con alta probabilidad de ser sustituidas, siendo que de las 4 posiciones que se identificaban en el período 2003-2009 en esta categoría, dos pasaron a ser superavitarias, una de probabilidad moderada de sustitución y la otra, con nula probabilidad. En cambio, sí se observan posiciones con probabilidad moderada y potencial. Hay tres posiciones con probabilidad moderada, que suman un déficit promedio en 2010-2015 de US\$ 32 millones, y otras cuatro posiciones con menor probabilidad (potencial), que representan un resultado negativo de US\$ 17 millones. $\mathrm{Si}$ efectivamente se sustituyeran la totalidad de las importaciones de las posiciones con alta, moderada o potencial probabilidad de sustitución, el saldo del sector biofarmacéutico podría reducirse en solo un $10 \%$ del déficit total (considerando los datos de comercio del subperíodo más reciente).

Cuadro Nro 1. Biofármacos: posibilidad de sustitución de importaciones ( $\mathrm{N}^{\circ}$ de posiciones y millones de U\$S dólares)

\begin{tabular}{|c|c|c|c|c|c|c|c|}
\hline \multirow{2}{*}{\multicolumn{2}{|c|}{ Probabilidad de sustitución Importaciones }} & \multicolumn{3}{|c|}{$\mathrm{N}^{\circ}$ Posiciones (NCM 12 digitos) } & \multicolumn{2}{|c|}{$\begin{array}{l}\text { Saldo Comercial anual promedio (en miles de } \\
\text { USS) }\end{array}$} & \multirow{2}{*}{ Moleculas representativas } \\
\hline & & $1998-2003$ & 2004-2009 & 2010-2015 & 1998-2003 & $2010-2015$ & \\
\hline Superavitarios & saldo comercial >0 & 7 & 9 & 13 & 16.401 & 101.557 & $\begin{array}{l}\text { Biológicos extractivos } \\
\text { (gonadotropinas, antiofídicos), } \\
\text { EPO, interferon alfa, filgrastim, }\end{array}$ \\
\hline $\begin{array}{l}\text { Alta probabilidad SI } \\
\text { (Deficitario pero existen } \\
\text { exportaciones intensas) }\end{array}$ & $\begin{array}{l}\text { Indice de Guber } \\
\text { Lloyd de } 1 \text { a } 0,66\end{array}$ & 2 & 4 & 2 & -3.106 & -10.523 & Interferon alfa, AC extractivos \\
\hline $\begin{array}{l}\text { Moderada prob de SI } \\
\text { (Deficitario pero existen } \\
\text { exportaciones } \\
\text { modoradac) }\end{array}$ & $\begin{array}{l}\text { Indice de Guber } \\
\text { Lloyd de } 0,66 \text { a } 0,33\end{array}$ & 7 & 3 & 3 & -12.323 & -32.863 & Insulina, interferon beta \\
\hline $\begin{array}{l}\text { Potencial prob de SI } \\
\text { (Deficitario y existen } \\
\text { exportaciones poco } \\
\text { sicinificativas) }\end{array}$ & $\begin{array}{l}\text { Indice de Guber } \\
\text { Lloyd de } 0,33 \text { a } 0,10\end{array}$ & 7 & 9 & 4 & -12.733 & -17.164 & $\begin{array}{l}\text { Reactivos de diagnóstico, } \\
\text { somatotropina, vacunas ADNr }\end{array}$ \\
\hline $\begin{array}{l}\text { Baja Probabilidad SI (no } \\
\text { existen exportadores o } \\
\text { lo hacen de forma } \\
\text { exigua) }\end{array}$ & $\begin{array}{l}\text { Indice de Guber } \\
\text { Lloyd de } 0,10 \text { a } 0\end{array}$ & 20 & 18 & 21 & -21.152 & -519.385 & $\begin{array}{l}\text { Etanercept (DCI); gemtuzumab; } \\
\text { Abciximab; alfa1-antitripsina; } \\
\text { basiliximab; infliximab, los } \\
\text { demas AMC, factor VIII } \\
\text { recombinante }\end{array}$ \\
\hline Total & & 43 & 43 & 43 & -32.913 & -478.377 & \\
\hline
\end{tabular}

Fuente: Elaboración en base a datos de Aduana, AFIP

(6) Esta metodología fue utilizada para estudios de diversos sectores, desde el metal-mecánico hasta el electrónico en la región de Tierra del Fuego (Peirano, 2013; Schorr y Porcelli, 2014). La misma es de carácter meramente exploratorio y su capacidad heurística depende del grado de desagregación de las posiciones arancelarias involucradas. 
Por último, se identifican un total de 21 posiciones en el último subperíodo con baja probabilidad de ser sustituidas, que registran exportaciones insignificantes pero abultadas importaciones, por lo que el déficit promedio resulta muy relevante. Puede apreciarse que si bien en el período 2004-2009 el número de posiciones con baja probabilidad de sustitución habían disminuido, como resultado del catching up tecnológico en las moléculas importadas en ese momento, las mismas vuelven a crecer en cantidad de productos y en monto de déficit. Para el promedio 2010-2015 el déficit de este conjunto ha sido de US\$ 519 millones explicando el $90 \%$ del total de las posiciones deficitarias.

Cuando analizamos cuales son los productos que explican la probabilidad (o no) de sustitución de se confirman varias de las hipótesis que se expusieron en la sección previa. Tal como se observa en el cuadro $\mathrm{N}^{\circ}$ 2, las moléculas más complejas como los anticuerpos monoclonales no solo constituyen el grupo con la contribución más significativa al déficit comercial del sector sino que dicho déficit es el que muestra menor posibilidad de ser sustituido para las capacidades existentes en el año 2015. Existen en nuestro país inversiones en plantas y su puesta en producción para el mercado depende de pasos regulatorios que se están llevando adelante ${ }^{7}$. Las moléculas con potencial alto de sustitución son moléculas de la primera generación de biofármacos como las hormonas de crecimiento y moléculas extractivas, que representan solo el2\% del saldo deficitario. Existen moléculas con moderado potencial de sustitución que aún no se han comoditizado totalmente, como los interferones Beta $1 \mathrm{a} 1 \mathrm{~b}$ e insulinas que explican el 6\% del déficit.

Cuadro Nro 2. Composición del déficit comercial en el período 2010-2014

\begin{tabular}{|c|c|c|c|c|c|}
\hline Grupo de productos & Pb. Alta & Pb Moderada & Pb. Potencial & Pb. Baja & Total \\
\hline Extractivos & $0,1 \%$ & $0,0 \%$ & $0,5 \%$ & $7,0 \%$ & $7,5 \%$ \\
\hline Filgrastim peguilado $\mathrm{ADNr}$ & $0,0 \%$ & $0,0 \%$ & $0,0 \%$ & $0,0 \%$ & $0,0 \%$ \\
\hline Interferon $\mathrm{ADNr}$ & $0,0 \%$ & $5,5 \%$ & $0,0 \%$ & $0,4 \%$ & $6,0 \%$ \\
\hline MABs ADNr & $0,0 \%$ & $0,0 \%$ & $0,0 \%$ & $40,4 \%$ & $40,4 \%$ \\
\hline Otros $\mathrm{ADNr} 2^{\circ}$ & $0,0 \%$ & $0,0 \%$ & $0,0 \%$ & $15,8 \%$ & $15,8 \%$ \\
\hline Reactivos & $0,0 \%$ & $0,0 \%$ & $2,5 \%$ & $0,2 \%$ & $2,7 \%$ \\
\hline Somatotropina y analogos & $1,7 \%$ & $0,0 \%$ & $0,0 \%$ & $0,0 \%$ & $1,7 \%$ \\
\hline Vacuna ADNr & $0,0 \%$ & $0,0 \%$ & $0,0 \%$ & $0,4 \%$ & $0,4 \%$ \\
\hline
\end{tabular}

Fuente: elaboración propia en base a datos de comercio INDEC

Nota: se incluye solo los grupos deficitarios.

Por tanto, se evidencia que de un total de 43 posiciones que corresponden a productos biofarmacéuticos, existen 13 que son superavitarias y que han mejorado el saldo comercial a lo largo de los últimos 16 años; un grupo de 5 posiciones, que sí podrían revertir la situación actual, comenzando a sustituir importaciones y mejorar su balance comercial; sin embargo aún existen 21 posiciones, que explican el crecimiento del déficit del sector y que dada la capacidad tecnológica y de producción hoy instalada presentan escasa probabilidad de ser sustituidas. $\mathrm{Al}$ analizar cuáles son los productos que explican la baja probabilidad de sustitución se corrobora que el déficit del sector se explica, principalmente, por los MABS, los cuales tienen un alto grado de complejidad de sus moléculas como también de los requerimientos de escala de producción y, por lo expuesto anteriormente, no cuentan aún con posibilidades en el corto plazo derevertir las importaciones (según la dinámica comercial).
Existen en este momento inversiones por parte de empresas locales buscando revertir esta situación para algunos productos - que están en proceso de validación de sus plantas por las autoridades regulatorias- y que aún no se reflejan en los datos comerciales (Gutman y Lavarello, 2015). En cambio, hay otros productos que actualmente presentan un saldo comercial deficitario pero que podrían comenzar a ser producidos con las capacidades actuales y, de esta manera, sustituirse importaciones, siendo que cuentan con una histórica trayectoria de exportaciones y, además, los requerimientos de escala no son altos y las moléculas tienen baja complejidad ${ }^{8}$.

(7) Dicha escasa probabilidad de sustituir importaciones es posible que pueda revertirse la en los próximos años en caso que maduren las inversiones realizadas por una importante firma local en el desarrollo del Rituximab y se aprueben moléculas actualmente en estudio por la autoridad regulatoria como es el caso del Etanercept. (8) En este ámbito, se encuentran algunas Vacunas con ADN recombinante, hormonas de crecimiento, interferones beta $1^{\mathrm{a}}$ y $1 \mathrm{~b}$, interferones alfa peguilados, interferones gamma e insulina. 


\section{Conclusiones y futuros interrogantes de investigación}

En los últimos quince 20 años un acotado conjunto de empresas argentinas ha logrado insertarse como exportadoras de moléculas biofarmacéuticas. No obstante, su participación en el mercado interno es secundaria. Si bien sus empresas se han insertado como exportadoras en ciertas moléculas extractivas y de primera generación, el déficit comercial es creciente y se explica por las moléculas de segunda generación (MABS). El fuerte crecimiento de las ventas internas de estos productos ha sido absorbido por las ventas de las grandes empresas líderes multinacionales que importan o la especialidad medicinal o los principios activos.

Esta situación plantea a las firmas locales el desafío de diversificarse hacia este segmento o enfrentar una situación en la que van a perder partes de mercado en el mercado farmacéutico global. Dadas las capacidades tecnológicas disponibles en términos de experiencia productiva las empresas cuentan con posibilidades de sustituir importaciones. No obstante, la tecnología no es estática sino que va mutando a lo largo de la difusión del paradigma: la entrada en los mercados de productos de la segunda generación implica mayores umbrales mínimos de escala y exigencias regulatorias que implican un gran salto para el sector.

La industria local cuenta con la posibilidad de avanzar hacia una segunda generación dado cuenta con la ventaja de haber alcanzado un umbral mínimo de conocimiento financiado por políticas de CyT, aprendizajes en la producción incorporadas en las rutinas de una base empresarial que supo ampliarse en los últimos años, y un status regulatorio que al igual que los otros elementos no es fácil de encontrar en un país en desarrollo. Valorizar estas ventajas plantea como interrogante cual es el tipo de empresas que llevaron adelante las estrategias incipientes de manufactura de biosimilares y cuál es el tipo de políticas de sustitución de importaciones a llevar a cabo a fin que estas experiencias se consoliden y adquieran un umbral mínimo como ara pasar de ser empresas exportadoras de la primera generación a la segunda generación de biosimilares.

En particular cabe interrogarse si es conveniente replicar los esquemas de políticas horizontales de CyT que inspiraron a las autoridades de Politica Tecnológica en los últimos años o si el Estado debe coordinar distintas de manera deliberada las acciones selectivas en términos de generación de capacidades, control selectivo del mercado interno y governance de las empresas. En este sentido existen un conjunto de acciones que fueron aplicadas en las experiencias de sustitución de importaciones en industrias de alta tecnología como la de semiconductores en Asia cuya aplicabilidad podría ser discutida para el caso de las industrias biotecnológicas:

- Incentivos fiscales y crediticios orientados a incentivar la I+D y fundamentalmente la inversión en plantas manufactura con la escala necesaria para producir moléculas más complejas.

- Una gestión selectiva del mercado interno a partir de la compra gubernamental orientada a aquellos proyectos que hayan aumentado la escala local de producción y cumplan con requisitos regulatorios.
- Nuevas modalidades de protección a la industria infante diferentes de las tarifas arancelarias: una gestión estratégica de la propiedad intelectual en la que la altura innovativa requerida para patentar sea lo suficiente elevada como para no bloquear las innovaciones incrementales asi como un marco regulatorio que vaya acompañando a las empresas locales para que las mismas adquieran la expertise regulatoria necesaria para competir en los mercados internacionales.

Cabe interrogarse por último si una estrategia nacional de promoción de la industrial no requiere se combinada con una governance flexible de las empresas promocionadas tal que la presencia del Estado en la propiedad de los joint-ventures- o algún esquema de inversiones cruzadas entre empresas nacionales- posibilite un horizonte de inversión más largo y evite la venta de las capacidades a grupos extranjeros. Quizás de esta manera Argentina podría insertarse a partir de una estrategia autónoma en la emergente industria biotecnológica

\section{Bibliografía}

Amsden, A. (1985). The state and Taiwan's economic development. Bringing the state back in, 78-106. https://doi.org/10.1017/ cbo9780511628283.005

Amsden, A. (2004). La sustitución de importaciones en las industrias de alta tecnología: Raúl Prebisch renace en Asia. Revista de la Cepal, $\mathrm{N}^{\circ} 82$, El desarrollo económico en los albores del siglo XXI, Bogotá, Alfaomega. https://doi.org/10.18356/bd14cfc2-es

Amsden, A. H. (1992). Asia's next giant: South Korea and late industrialization. Oxford University Press. https://doi. org/10.1093/0195076036.001.0001

Cimoli, M., Dosi, G., Nelson, R., \& Stiglitz, J. (2006). Institutions and policies shaping industrial development: an introductory note. LEM Papers Series, 2, 2006-02. https://doi.org/10.1093/acprof:o so/9780199235261.003.0002

Dosi, G. (1988). Sources, procedures, and microeconomic effects of innovation.Journal of economic literature, 1120-1171. https://doi. org/10.12691/jbe-4-1-1

Gutman G. and Lavarello P. (2014b): Biotecnología Industrial en Argentina. Estrategias empresariales frente al nuevo paradigma, Letra Prima, CEUR-CONICET, CABA, E-Book. DOI: 10.13140/2.1.4480.1929

Gutman G., and Lavarello P. (2014 a) "Biopharmaceuticals and firm organization in Argentina.Opportunities and challenges", en International Journal of Technology and Globalisation, Elsevier.https://doi. org/10.1504/ijtg.2014.064740

Kim, L. (1990): Imitation to Innovation. The Dynamics of Korea's Technological Learning, HBS Press, https://doi.org/10.1215/s12280-007-9017-2

Lavarello, P. (2014). Convergencia de paradigmas biotecnológicos y estrategias de los grupos líderes mundiales. Problemas del desarrollo, 45(177), 9-35. https://doi.org/10.1016/s0301-7036(14)70861-2 
Malerba F, Nelson R (2011) "Learning and catching up in different sectoral systems: evidence from six industries" Industrial and Corporate Change (2011) 20 (6), pp. 1645-1675.Niosi, 2011. https://doi. org/10.1093/icc/dtr062

Moorkens, E., Jonker-Exler, C., Huys, I., Declerck, P., Simoens, S., \& Vulto, A. (2016). Overcoming barriers to the market access of biosimilars in the European Union: The case of biosimilar monoclonal antibodies. Frontiers in Pharmacology, 7, 193. https://doi.org/10.3389/ fphar.2016.00193

Ocampo, José Antonio (2009): Los paradigmas del desarrollo en la historia latinoamericana, Facultad de Ciencias Económicas y Administración, Universidad de la República, Montevideo. Authors: José Antonio Ocampo https://doi.org/10.18356/5e511b80-es

Peirano, F. (2013). El complejo productivo de bienes de capital. En Stumpo G. Y Rivas, D. : La industria argentina frente a los nuevos desafíos y oportunidades del siglo XXI. Santiago: CEPAL, 2013. p. 61100. LC/L. 3637.

Pérez, C. (2003). Technological change and opportunities for development as a moving target. Trade and Development: Directions for the 21st Century, 100. https://doi.org/10.4337/9781843767473.00010
Pérez, Carlota y Soete, Luc (1988) "Catching up in technology: Entry barriers and windows of opportunity”, G. Dosi y otros (ed.),Technical Change and Economic Theory, Londres, Pinter Publishers. doi:10.1007/BF01384909

Pisano, G. (2002). Pharmaceutical biotechnology.Technological innovation and economic performance, 347-365. https://doi.org/10.1017/ CBO9781139175128

Rader, R. A. (2013). An analysis of the US biosimilars development pipeline and likely market evolution. BioProcess Int, 11(6). https:// doi.org/10.12665/j124.rader

Ray, D. (1998). Development economics. Princeton University Press. https://doi.org/10.1057/9780230226203.0385

Schorr, M., \&Porcelli, L. (2014). La industria electrónica de consumo en Tierra del Fuego. Régimen promocional, perfil de especialización y alternativas de desarrollo sectorial en la posconvertibilidad. IDAES/ UNSAM, Documentos de Investigación Social, (26).

Thorp, R., et al. "Progreso, pobreza y exclusión: una historia económica de América Latina en el siglo XX". Econometric Society, Evanston, Ill.(EUA)., 1998. https://doi.org/10.1057/9780230595682

Vernon, R. (1966). International investment and international trade in the product cycle. The quarterly journal of economics, 190-207. https://doi.org/10.1002/tie.5060080409 